\title{
A New Solid Boundary Treatment Algorithm For Smoothed Particle Hydrodynamics
}

\author{
M. B. Liu*, J. R. Shao \\ Institute of Mechanics, Chinese Academy of Science, Beijing 100190, China
}

Email: liumoubin@imech.ac.cn

\begin{abstract}
This paper presents a new solid boundary treatment algorithm for smoothed particle hydrodynamics (SPH). Two types of virtual particles are used to model the solid boundary, and information of the virtual particles can be obtained using enhanced SPH approximations from fluid particles. Numerical examples demonstrate that SPH with this new solid boundary algorithm can lead to smooth and accurate pressure field, even for complex solid boundaries.
\end{abstract}

Key words: solid boundary, fixed particle, flow pattern, SPH

\section{INTRODUCTION}

Smoothed particle hydrodynamics is a popular meshfree, Lagrangian, particle method with some attractive features $[1,2]$, and it can naturally obtain time history of the material particles, and deal with large deformations easily. However, further developments and applications of SPH have been hindered by the solid boundary treatment, which is a key factor influencing the accuracy of SPH.

There are different efforts to treat solid boundaries in SPH, and so far none is satisfactory considering accuracy, efficiency, and flexibility. Monaghan [3] used a line of fixed particles to model the solid boundary, and the fixed particles can exert repulsive force on approach fluid particles to prevent them from penetration. The approach usually leads to large pressure oscillations in the solid boundary area. Libersky and Petschek [4] first introduced ghost particles to reflect a symmetrical surface boundary condition, while it can only deal with simple boundary conditions. Takeda [5] and Morris [6] also conducted works on solid boundary treatment, and their improvements are not always effective, especially for complex solid boundaries.

\section{A NEW SOLID BOUNDARY TREATMENT ALGORITHM}

In this paper, we propose a new solid boundary treatment algorithm with two types of virtual particles, repulsive particles (virtual particles of Type 1) right on the solid boundary, and ghost particles (virtual particles of Type 2) in the solid boundary area within the support domain of the smoothing function from the solid boundary (Figure 1). The novelty of the algorithm include (1) an improved repulsive force for repulsive particles, and (2) an improved interpolation scheme to approximate the information of the virtual particles.

Firstly, the repulsive particles exert a distance-dependent repulsive force on fluid particles to prevent them from unphysical penetration. The improved repulsive force is as follows

$F_{i j}=\frac{0.01 c^{2}}{y} \chi f(q) \frac{x_{i j}}{r_{i j}}, q=y /\left(0.75 h_{i j}\right), \chi=1-\frac{x}{\Delta d}, 0<x<\Delta d, f(q)= \begin{cases}2 / 3, & 0<q \leq 2 / 3 \\ \left(2 q-1.5 q^{2}\right), & 2 / 3<q \leq 1 \\ 0.5(2-q)^{2}, & 1<q<2 \\ 0, & \text { otherwise }\end{cases}$

where $x, y$ is the horizontal and vertical distance between two adjacent particles, $\Delta d$ is the initial particle spacing. 


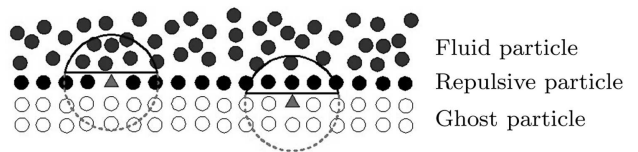

Figure 1: Illustration of the new solid boundary algorithm

Secondly, the information of the virtual particles can be obtained from approximating the corresponding field variables from fluid particles within the support domain of the corresponding virtual particle (either Type 1 or Type 2), with enhancement using Shepard Filter method or moving least square method, which are usually used only for approximating field properties of fluid particles.

\section{NUMERICAL EXAMPLES}

In this section, two numerical examples are presented to demonstrate the effectiveness of the new solid boundary treatment algorithm. The first example involves dam break against a vertical wall (Figure 2(a)), and in the second example (Figure 2(b)), a sharp-edged obstacle is placed before the vertical wall during the dam breaking process.

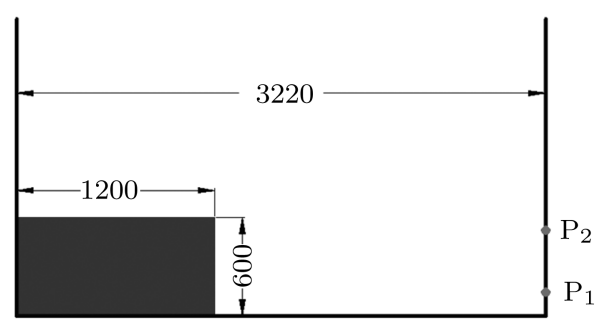

(a) Dam-break flow against a vertical wall

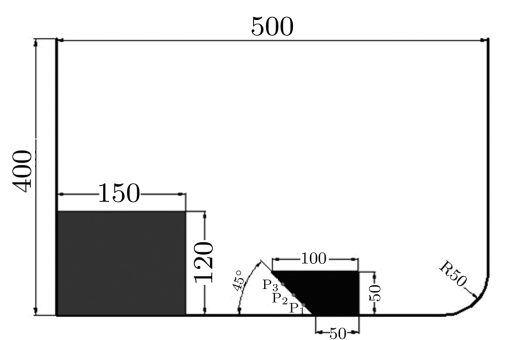

(b) Dam-break flow with a sharp-edged obstacle

Figure 2: Dam-break flows against a vertical wall and with a sharp-edged obstacle.

1. Free dam break against a vertical wall The free dam break against a vertical wall has been modeled using the SPH method by a number of researchers. Conventional SPH method can also capture the flow behavior of dam-break such as water height and surge front. However, Conventional SPH is not able to give precise prediction of the pressure load on the solid boundary as it can not resolve smooth and accurate pressure field, especially near the solid boundary area.

Figure 3 shows the particle distribution and pressure field obtained using the repulsive force boundary algorithm [3], and the new algorithm. It is clear that the repulsive force boundary algorithm leads to rough pressure field with numerical oscillations. It is therefore difficult to obtain accurate pressure loads on the solid surface. In contrast, the new solid boundary treatment algorithm leads to much smoother pressure field, and more ordered particle distribution. The large impact pressure in the corner area can be effectively predicted with obvious pressure layers.

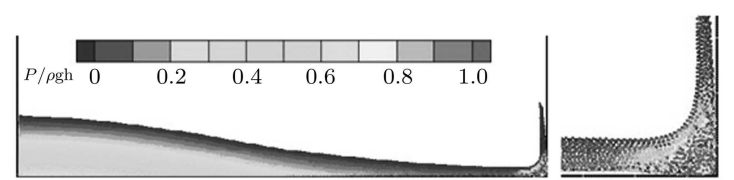

(a) Using the repulsive force boundary algorithm

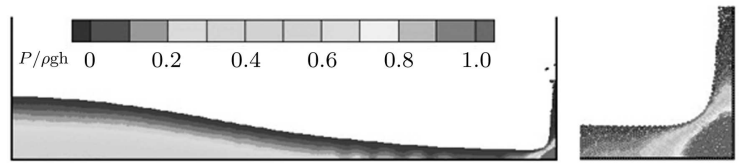

(b) Using new algorithm

Figure 3: Particle distribution and pressure field obtained using the repulsive force boundary algorithm, and the new algorithm, with zoom-in plots at the corner areas

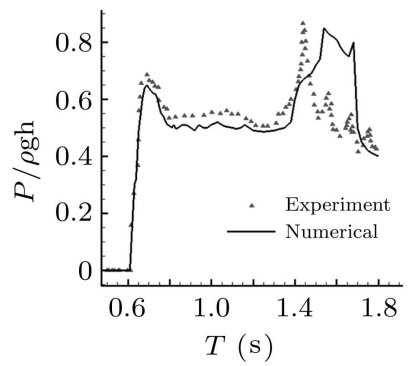

(a) Pressure load on $\mathrm{P}_{1}$

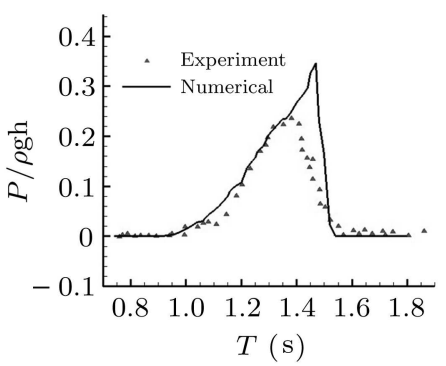

(b) Pressure load on $\mathrm{P}_{2}$

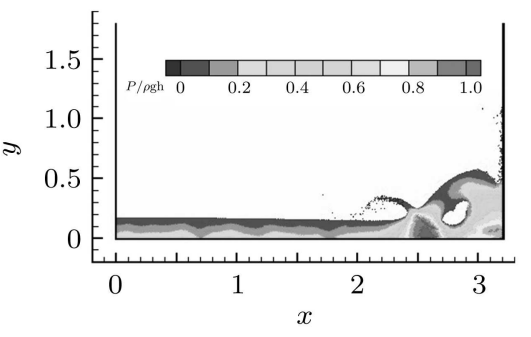

(c) Pressure load at $1.6 \mathrm{~s}$

Figure 4: The pressure load on two probe points (P1 and P2) on the vertical wall, and pressure field at $1.6 \mathrm{~s}$

To quantitatively validate the pressure load on the solid boundary, two probe points (see Figure 2(a)) are used, and the pressures on P1 (Figure 4(a)) and P2 (Figure 4(b)) obtained using the new boundary algorithm are compared 
with the experiment observations by Buchner [7]. At approximately $0.6 \mathrm{~S}$, the water front impacts onto point P1, which generates a big pressure jump (approximately 0.65 times of the maximum hydrostatic pressure). From 0.6 to $1.4 \mathrm{~s}$ the pressure reduces to smaller amplitude. It is obvious that before $1.4 \mathrm{~s}$, the numerical results agree well with experimental observations. While after $1.4 \mathrm{~s}$, there exists bigger differences, largely due to the air entrapment (cavity) in the simulation. It is expected to obtain better agreement if using two phase model (water and air) in the SPH simulation. It is noted that even for later stages, the obtained pressure field (see Figure 4(c)) is also very smooth.

2. Free dam break against a sharp-edged obstacle This example is similar to the above case except that a sharpedged obstacle is placed before the right vertical wall. The sharp-edged obstacle can lead to a very big impact during the dam breaking process. After the water front impacts onto the sharp edge, water particles will be spread away from the edge, and generates a long strip of water. Most importantly, some particles splash away from the water strip and then fall onto the bulky water, which leads to transient heavy pressure in some areas of the bulky water. Using conventional SPH method with previously boundary algorithms is not able to accurately resolve the pressure field near the sharp edge, and near areas where splashed particles fall on the bulky water. Again the new solid boundary algorithm can obtain smooth pressure field, well capture the spreading and formation of water strip. The flow patterns agree with those obtained by Colicchio [8]. And the pressures on a probe point (P1, see Figure 2) also agree well with the results by Marrone [9] who modeled the same case using fixed ghost method with much more particles and much larger computational cost.

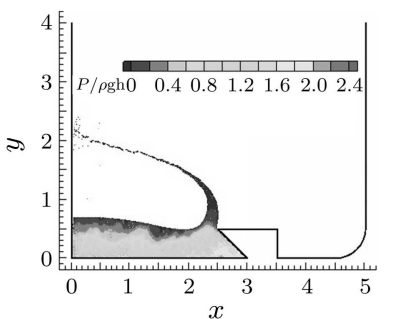

(a) $0.8 \mathrm{~s}$

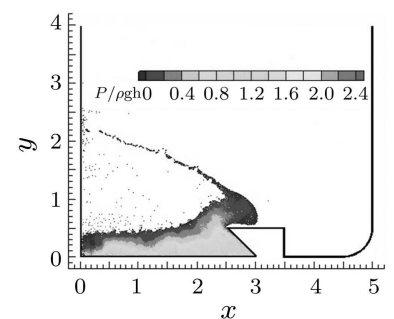

(b) $1.31 \mathrm{~s}$

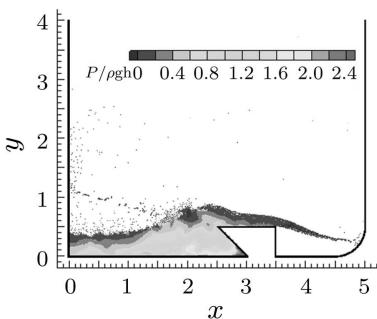

(c) $1.67 \mathrm{~s}$

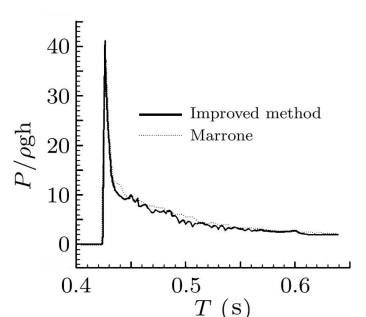

(d) Comparison of the pressure in P1

Figure 5: The pressure field in $0.8 \mathrm{~s}, 1.31 \mathrm{~s}, 1.67 \mathrm{~s}$ and the comparison of the pressure in P1

\section{CONCLUSIONS}

A new solid boundary treatment algorithm is introduced in this paper to simulate violent free surface flows. The algorithm consists an improved repulsive force, and an enhancement on virtual particle information approximation. The effectiveness of the new solid boundary treatment algorithm has been demonstrated by two numerical examples with better accuracy and smoother pressure field.

\section{REFERENCES}

1 Gingold RA, Monaghan JJ. Smoothed particle hydrodynamics-theory and application to non-spherical stars. Monthly Notices of the Royal Astronomical Society, 1977; 181: 375-389

2 Liu M, Liu G. Smoothed particle hydrodynamics (SPH): An overview and recent developments. Archives of Computational Methods in Engineering, 2010; 17(1): 25-76

3 Monaghan J. Simulating free surface flows with SPH. Journal of Computational Physics, 1994; 110: 399-399

4 Libersky LD, Petschek AG, Carney TC, et al. High-strain lagrangian hydrodynamics - a 3-dimensional SPH code for dynamic material response. Journal of Computational Physics, 1993; 109(1): 67-75

5 Takeda H, Miyama S, Sekiya M. Numerical simulation of viscous flow by smoothed particle hydrodynamics. Progress of Theoretical Physics, 1994; 92(5): 939-960

6 Morris J, Fox P, Zhu Y. Modeling low reynolds number incompressible flows using SPH. Journal of Computational Physics, 1997; 136(1): 214-226

7 Buchner B. Green water on ship-type offshore structures. [PhD Thesis], Delft University of Technology, 2002

8 Colicchio G. Violent disturbance and fragmentation of free surfaces. [PhD Thesis], University of Southampton, 2004

9 Marrone S, Antuono M, Colagrossi A, et al. $\delta$-SPH model for simulating violent impact flows. 2010. (Personal Communications) 